\title{
Harmonizing MAC and Routing in Low Power and Lossy Networks
}

\author{
Piergiuseppe Di Marco ${ }^{\dagger}$, Carlo Fischione ${ }^{\dagger}$, George Athanasiou ${ }^{\dagger}$, and Prodromos-Vasileios Mekikis ${ }^{\ddagger}$
}

\begin{abstract}
Medium access control (MAC) and routing protocols are fundamental blocks in the design of low power and lossy networks (LLNs). As new networking standards are being proposed and different existing research solutions patched, evaluating the performance of the network becomes challenging. Specific solutions that can be individually efficient, when stacked together may have unexpected effects on the overall network behavior. In this paper, we provide an analysis of the fundamental MAC and routing protocols for LLNs: IEEE 802.15.4 MAC and IETF RPL. Moreover, a characterization of their crosslayer interactions is presented by a mathematical description, which is essential to truly understand the protocols mutual effects and their dynamics. Novel metrics that guide the interaction between MAC and routing are compared to existing metrics. Furthermore, a protocol selection mechanism is implemented to select the appropriate routing metric and MAC parameters given specific performance constraints. Analytical and experimental results show that the behavior of the MAC protocol can hurt the performance of the routing protocol and vice versa, unless these two are carefully optimized together.
\end{abstract}

\section{INTRODUCTION}

Research communities and standardization bodies have been working in the recent years for the definition of the communication infrastructure for low power and lossy networks (LLNs) [1]. An LLN is composed of many embedded devices with potentially limited power, memory, and processing resources wirelessly interconnected. A variety of applications is envisioned thanks to protocol inter-operability between different devices that are part of LLNs, including building and industrial automation, health care, smart grids, and security. To support such an increasing number of applications, wireless MAC and routing protocols must be inherently efficient, scalable, and inter-operable.

The IEEE 802.15.4 [2] standard defines flexible physical and MAC layers for low data rate and low power applications. The standard has been adopted with some modification also by a number of other solutions and according to recent surveys, it already represents more than $50 \%$ of building and industrial automation market [3]. Meanwhile, routing protocols are still being under standardization. The Internet Engineering Task Force (IETF) is currently working towards the specification of a reference standard for LLNs, the IETF routing protocol for low power and lossy networks (RPL) [4]. RPL is designed to

$\dagger$ P. Di Marco, C. Fischione, and G. Athanasiou are with the ACCESS Linnaeus Centre, Royal Institute of Technology, Stockholm, Sweden. E-mails: \{pidm, carlofi, georgioa\}dee.kth.se.

$\ddagger$ P. Mekikis is with the Signal Theory and Communications Department, Technical University of Catalonia, Barcelona, Spain. E-mail: vmekikisetsc.upc.edu. P. Mekikis was with the ACCESS Linnaeus Centre when contributing to this work.

The work was supported by the EU projects Hycon2, Hydrobionets and the Swedish Research Council. be compatible with many existing MAC protocols, particularly the IEEE 802.15.4 MAC.

In many applications, it is inefficient to design MAC and routing protocols separately. In process control, the state information must be transmitted to the controller over a network guaranteeing a certain reliability and delay. In the network, the decision over different routing paths depends on the performance indicators (successful packet reception probability, or reliability, delay, and energy consumption), which are influenced by the MAC parameters.

In this paper, we first emphasize the main characteristics of the IEEE 802.15.4 MAC protocol and the IETF RPL. We provide a mathematical description to characterize the protocols mutual effects and their dynamics. In particular, we show that the level of contention at the MAC layer influences the routing decisions in an unexpected manner. In addition, we prove that, in the presence of dominant paths in the network, the performance indicators at MAC layer are significantly affected. We propose metrics that guide the interactions between MAC and routing, and we introduce a mechanism that selects the appropriate routing metric and adapts the corresponding MAC parameters to minimize the energy consumption, given reliability and delay constraints. We experimentally evaluate a) the performance of the proposed metrics through the comparison to existing metrics, and $b$ ) the effectiveness of the protocol selection mechanism. Moreover, the study is validated by experiments in realistic environments.

The rest of the paper is organized as follows. In Section II, we give an overview of the basic functionalities/parameters of IEEE 802.15.4 MAC and IETF RPL. In Section III, we motivate our analysis and emphasize the importance of crosslayer interactions in LLNs. In Section IV, the interactions among MAC and routing are modeled. Section V follows the guidelines defined in the previous section and defines macaware routing metrics. In Section VI, we present the experimental evaluation of the proposed metrics. Finally, Section VII concludes the paper.

\section{IEEE 802.15.4 MAC AND RPL}

In this section, we give an overview of the IEEE 802.15.4 MAC and RPL protocols. Moreover, we present representative analytical and experimental studies in literature.

\section{A. IEEE 802.15.4 MAC Protocol}

The IEEE 802.15.4 MAC defines two basic access modalities: contention-based MAC, with a simple unslotted carrier sense multiple access collision avoidance (CSMA/CA), and hybrid-based MAC with a slotted CSMA/CA and a contentionfree operation based on guaranteed time slot (GTS) allocation. 
In the following, we focus on the unslotted modality, which is of major interest for RPL.

Consider node $V_{i}$ trying to transmit a packet. First, the node waits for a random number of time units in the window $\left[0-2^{m_{0}}\right]$. Then, the node performs a clear channel assessment (CCA). If the channel is idle, the node begins the packet transmission. In case that the CCA fails due to busy channel, the MAC layer increases the backoff window exponentially. We indicate by $\alpha_{i}$ the probability of busy channel for node $V_{i}$. If the backoff exponent reaches a maximum value $m_{b}$, it remains at that value until it is reset. If the number of backoffs exceeds a maximum number $m$, then the packet is discarded due to channel access failures. The reception of an acknowledgement (ACK) is interpreted as successful packet transmission. If the node fails to receive the ACK, the MAC re-initializes the backoff window and follows the CSMA/CA mechanism to re-access the channel. After a maximum number of retransmissions $n$, the packet is discarded.

An adaptation mechanism of the IEEE 802.15.4 MAC has been proposed in [5], and experimentally validated on a wireless body sensor network for medical application. However, the approach is not based on analytical modeling. The importance of a mathematical modeling of MAC protocol has been advocated in recent literature [6], [7]. In [6], a Markov chain model approach is used to provide a distributed adaptive algorithm for minimizing the power consumption of single hop star networks using the CAP of the IEEE 802.15.4 MAC, while guaranteeing a given successful packet reception probability and delay constraints. In [7], an automatic MAC protocol selection is proposed. The idea is to provide a mathematical analysis of various MAC protocols and to choose the optimal MAC and optimal parameters for the selected MAC modality, topology, and packet generation rate. The main value of these approaches is that the algorithms do not require any modification of the IEEE 802.15.4 standard and could be easily implemented on top of the current network device implementation. However, their application is limited to single-hop networks. A framework for MAC parameter adaptation based on analytical modeling has been also presented in [8]. The approach has been developed for XMAC and LPP protocol. However, the results are not directly applicable to the IEEE 802.15.4 MAC due to the different channel access mechanism.

\section{B. Routing Protocol for Low Power and Lossy Networks}

RPL constructs destination-oriented directed acyclic graphs (DODAGs) over the network, according to optimization objectives. Every node in a DODAG is identified by a rank, a scalar value that represents the relative position of the node with respect to other nodes and the DODAG root. Nodes build and maintain DODAGs by periodically multicasting messages, called DODAG information object (DIO), to their neighbors. To join a DODAG, a node listens to the DIO messages sent by its neighbors, selects a subset of these nodes as its parents, and compute its rank. Although the rank is computed by using link costs, topology building and maintenance mechanisms can be made independently of packet forwarding procedures.

In Fig. 1, we visualize a DODAG example that we use as reference topology in our evaluation study. Each end-device has one or more nodes in its parent set. The packet forwarding

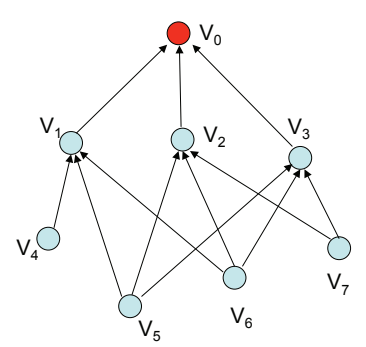

Fig. 1. DODAG example for a topology with single root node $V_{0}$.

is based on end-to-end metrics and application constraints. There are various metrics and constraints that can be used. Reliability, packet delay, and node energy consumption are indicators that can be used both as metrics and constraints. Expected transmissions count (ETX) [9] is a reliability metric that indicates the number of retransmissions a node expects to execute to successfully deliver a packet to the destination node. For example in Fig. 1, we consider node $V_{5}$ that has $V_{1}$, $V_{2}$, and $V_{3}$ in its parent set. The metric of a generic link $(i, j)$ is denoted as $\pi_{i, j}$. Then, $V_{5}$ chooses $V_{1}$ as next hop node if $\pi_{5,1}+\pi_{1,0}$ outperforms $\pi_{5,2}+\pi_{2,0}$ and $\pi_{5,3}+\pi_{3,0}$.

In [10], an experimental performance evaluation of RPL that uses the hop count metric and the ETX metric is presented. The authors discuss the stability of the network and the DODAG. However, the study does not consider the combined effect of a contention-based MAC protocol and the routing metrics. In the back-pressure collection protocol (BCP) [11], an extension of the ETX metric is proposed by introducing a dynamic back-pressure routing metric. In $\mathrm{BCP}$, the routing and forwarding decisions are made on a per-packet basis by computing a back-pressure weight of each outgoing link that is a function of node queues and link state information. BCP is tested over a low power contention-based MAC, but it does not account explicitly for the effects of limited number of backoffs and retransmissions in the IEEE 802.15.4 standard. In [12], the authors propose a metric for opportunistic routing for very low-duty cycled MACs that considers the expected number of wake-ups that are required to successfully deliver a packet from source to destination. In [13], a multi-path opportunistic routing is proposed for time-constrained operations over IEEE 802.15.4 MAC. However, load balancing and the effects of contention-based access are not considered. A study on the interaction of RPL with the MAC layer is presented in [14]. The authors investigate the use of a receiver-initiated MAC protocol in enhancing the performance of RPL. However, the work in [14] does not include the specifications of the IEEE 802.15.4 MAC. We provide details of the MAC-routing interaction in the next section.

\section{MAC AND ROUTING INTERACTIONS}

In this section, we describe the interactions between MAC and routing through the feedback loop visualized in Fig. 2 and we highlight the importance of cross-layer interactions in the LLNs standards.

The supported application sets a traffic generation rate for each node in the network which is related to the required sampling time of the sensing operation in case of sensor networks, or a generic data generation rate of the application. Moreover, 


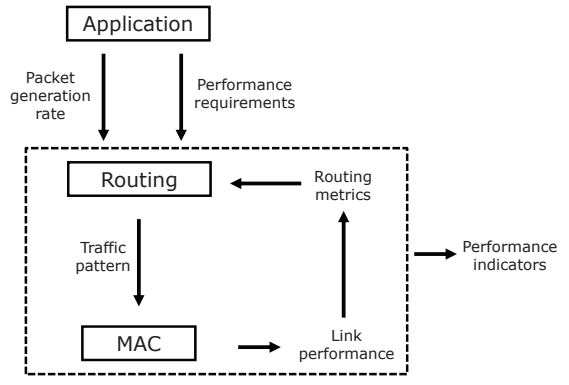

Fig. 2. The loop of MAC and routing interactions. The closed loop can lead to instabilities if these interactions are not considered when selecting the protocols.

the application layer determines performance requirements for the lower protocol layers (e.g., minimum data delivery rate and maximum packet delay). The routing layer combines the topological information in a network communication graph. In addition, based on specific metrics, the routing protocol takes appropriate decisions to distribute the traffic in the network. It affects the load that each link, regulated by the $M A C$ layer, has to serve. As an output of the MAC process, we get the link performance in terms of energy consumption, reliability, or delay. Furthermore, the link performance indicators may influence directly the routing metric, so closing the loop between MAC and routing layers. The combined analysis of the MAC and the routing layers determines the end-to-end performance indicators.

Due to the inter-dependency, if the routing is designed without taking into account the MAC, the end-to-end performance indicators could be far from desired by the application. By extending the approach introduced in [7], we propose a mathematical selection tool based on a library of compatible MAC and routing protocols. The tool picks the best protocol solution in the library, with optimal parameters for the specific application. The selection takes as input the network topology and packet generation rate, the application requirements for reliability, delay, and energy consumption, and the resource constraints from the underlying physical layer. The idea of analytical description and optimization of the cross layer interactions is not new, but as a matter of fact, the standardization process seems to consider it partially.

\section{JOInt MAC And Routing Model}

This section describes the joint model of the IEEE 802.15.4 MAC and the IETF RPL.

As introduced in Section II-A, the level of contention at node $V_{i}$ is described by the busy channel probability $\alpha_{i}$, which is a function of the MAC parameters $\left(m_{0}, m_{b}, m\right.$, and $\left.n\right)$, of the traffic $Q_{i}$, and of the busy channel probabilities of the neighboring nodes $\alpha_{k}$. An analytical model based on Markov chain analysis to derive $\alpha_{i}$ analytically is presented in [15]. To provide a simple solution, we assume that $\alpha_{i}$ is estimated at node $V_{i}$ during the CCA. The busy channel probability is initialized at the beginning of the node's operation. The estimation of the probability uses a sliding window. When the node senses the channel, the probability is updated by $\alpha_{i}=r \alpha_{i}+(1-r) \hat{\alpha}_{i}$ for some $r \in(0,1)$, respectively. Note that $\hat{\alpha}_{i}$ is the busy channel probability of the current sliding window.
Given that the channel is idle during CCA, the probability $\gamma_{i, j}$ that a packet is not received at destination is given by

$$
\gamma_{i, j}=p_{\mathrm{coll}, i}+\left(1-p_{\mathrm{coll}, i}\right) p_{i, j}
$$

where $p_{\mathrm{coll}, i}$ is the probability that $V_{i}$ encounters a collision due to a simultaneous transmission as the probability that at least another node performs the CCA and finds the channel idle in the same time unit, namely $p_{\text {coll }, i}=\alpha_{i} / T_{s}$. The bad channel probability $p_{i, j}$ is the probability that the link quality is not sufficient to yield a successful packet reception at the receiver $V_{j}$. We notice that the probability $\gamma_{i, j}$ is an upper bound of the packet loss probability since it considers unsuccessful all the events in which at least another node is transmitting, independently of the received SINR.

We evaluate the reliability as the successful packet reception rate or, equivalently, the complement of the discard probability. In the IEEE 802.15.4 CSMA/CA mechanism, packets are discarded due to the following reasons: (i) channel access failure or (ii) retransmissions limit. Channel access failure happens when a packet fails to obtain clear channel within the maximum number of backoffs $m$ (at any retransmission stage). Furthermore, a packet is discarded if the transmission fails due to repeated collisions and losses after the maximum number of retransmissions $n$. Therefore, the probability that the packet is discarded due to channel access failure is

$$
p_{i, j}^{c f}=\alpha_{i}^{m+1} \sum_{k=0}^{n}\left(\gamma_{i, j}\left(1-\alpha_{i}^{m+1}\right)\right)^{k} .
$$

The probability of a packet being discarded due to the retransmissions limit is

$$
p_{i, j}^{c r}=\left(\gamma_{i, j}\left(1-\alpha_{i}^{m+1}\right)\right)^{n+1} .
$$

The reliability of the link $(i, j)$ is given by

$$
R_{i, j}=1-p_{i, j}^{c f}-p_{i, j}^{c r}
$$

We notice that the reliability is dependent only on the busy channel probability $\alpha_{i}$, the bad channel probability $p_{i, j}$, and the MAC parameters $m, n$. As explained before, the busy channel probability $\alpha_{i}$ can be estimated at the transmitter during the CCA. Therefore, a node does not require any extra communication and sensing state to estimate this probability compared to the default IEEE 802.15.4 standard. As shown in [16], the bad channel probability $p_{i, j}$ can be retrieved by the link quality indicator (LQI) which is a parameter offered by the IEEE 802.15.4 physical layer header for every received packets [2].

We represent the dynamical interaction of MAC and routing using a statistical model. Let $\lambda_{j}$ be a vector of traffic generation rates, where each component is associated to a node of the network. In addition to $\lambda_{j}$, node $V_{j}$ has to forward traffic generated by its children. Let $Q_{j}$ be the traffic generated plus the traffic that the node has to forward (generated and routed by other nodes) to a node in the set of candidate receivers $\Gamma_{i}$.

RPL path selection is modeled by a real valued matrix $\mathbf{M}$, in which element $M_{i, j}$ corresponds to the probability that the metric $\pi_{i, j}$ evaluated for the link $(i, j)$ is the best among the set of candidate receivers $\Gamma_{i}$. 
The distribution of the traffic flows along the network is modeled by the matrix $\mathbf{M}$, and by a scaling due to that only successfully received packets are forwarded. Therefore, we define a matrix $\mathbf{T}$ such that $T_{i, j}=M_{i, j} R_{i, j}$ where $R_{i, j}$ is the reliability in the link $(i, j)$, which clearly depends on the traffic rate of the node. It follows that the vector of node traffic generation probabilities $Q$ is the solution of a system of flow balance equations $Q=Q \mathbf{T}+\lambda$. In steady state, we have

$$
Q=\lambda[\mathbf{I}-\mathbf{T}]^{-1},
$$

where $\mathbf{I}$ is the identity matrix and ${ }^{-1}$ denotes the matrix inversion, which always exists because $\mathbf{T}$ is a probability matrix and thus has eigenvalues strictly less than one. Eq. (IV.5) gives the fundamental relation between the packet generation rate, the effect of routing (via $\mathbf{M}$ ) and the performance of the MAC layer (via the link reliability $R_{i, j}$ ). Eq. (IV.5) together with the expressions for the per-link reliability $R_{i, j}$ gives the distribution of the traffic in the network. In practice, Eq. (IV.5) is the fundamental equation to model mathematically in a simple yet effective manner the joint effects of MAC and routing. The $i$-th component of the vector $Q$ is the amount of traffic that the $i$-th node has to forward to its parent per unit time. This traffic is handled by the MAC and thus determines the per-link performance such as reliability, delay and energy consumption. We remark that due to the acyclic structure of the communication graph, the elements $Q_{i}$ can be calculated locally at node $V_{i}$ by using information on the own traffic $\lambda_{i}$, and the estimate of the total coming traffic from children nodes. When node $V_{i}$ switches its selected parent to $V_{j}$, it combines the information on the traffic $Q_{j}$, which can be encapsulated in the DIO messages of $V_{j}$ and the forwarded traffic $V_{i}$. Therefore, we can define routing metrics that exploit our model to locally estimate the reliability and the effects of routing decisions on the traffic distribution.

\section{MAC-Aware Routing Metrics}

In this section, we present two metrics that are based on the link performance at MAC layer. Moreover, they are simple and easy-to-implement in practice using mechanisms defined in standards.

First, we introduce the $R$-metric. For node $V_{i}$, we define the metric $R(i)=R_{i, 0}$, where $R_{i, 0}$ is the end-to-end reliability between node $V_{i}$ and $V_{0}$. Then, nodes forward their packets by selecting a parent $V_{j}$ that

$$
\underset{j \in \Gamma_{i}}{\operatorname{maximize}} \quad R_{i, j} \cdot R(j) .
$$

The set of candidate receivers $\Gamma_{i}$ is composed by the set of nodes that can guarantee a progress towards the destination $V_{0}$, according to RPL specifications. The $R$-metric extends the ETX metric at the MAC layer, by considering also packet losses due to the MAC contention. In fact, the ETX is based on the expected number of retransmissions needed to reach a destination, by counting the number of transmissions and ACKs. ETX is an additive metric over the path. $R$-metric is based on the probability that a packet is correctly received in each link of the paths, within a maximum number of backoffs and retransmissions at the MAC layer. We illustrate the difference between the two metrics through a simple numerical example.

Consider the multi-hop topology in Fig. 1. Node $V_{7}$ has two paths to the destination, one path through $V_{2}$ and the other through $V_{3}$. Assume that the path through $V_{2}$ has $E T X_{7,2}=2.1$ and $E T X_{2,0}=2.1$, which determines a total expected number of retransmissions $E T X_{7,2,0}=4.2$ to the destination. The second path has $E T X_{7,3}=1.1$ and $E T X_{3,0}=2.9$, which makes a total $E T X_{7,3,0}=4.0$. In absence of a retry limit at MAC layer, the second path through $V_{3}$ has the minimum ETX value and gives the highest endto-end delivery ratio. However, if we set a maximum number of retransmissions $n=4$, as specified by the IEEE 802.15.4 standard, and by assuming independent loss probability for consecutive retransmissions, the end-to-end success rate is $92.3 \%$ in the path through $V_{2}$, while only $87.7 \%$ in the path through $V_{3}$, due to higher packet loss probability in the link $(3,0)$. The path through $V_{2}$ has a $5 \%$ worse ETX value but it guarantees a $5 \%$ better end-to-end reliability, and this effect is expected to be more evident by considering correlation between consecutive retransmissions.

Moreover, the $R$-metric extends the concept of link quality, by including the effects of contention at the MAC layer. In fact, even in case of same LQI indicator among different links, the routing decision determines a different distribution of the traffic over the network and a different level of contention at MAC layer for the forwarding nodes, thus different busy channel probabilities $\alpha_{i}$, which are included in the expression of the reliability. We recall that the estimation of the busy channel probability can be performed at the node without extra information needed or modification to the standard IEEE 802.15.4 MAC. Moreover, its estimation is faster than the ETX estimation, which is performed over a certain number of received ACKs.

For low power applications the reliability can be just set in terms of minimum requirement, and the objective is mainly the network lifetime. We then propose a metric called $Q$ metric, which distributes the forwarded traffic to provide load balancing in the network. In particular, the $Q$-metric at nodes $V_{i}$ computes the traffic $Q_{i}$. Node $V_{i}$ selects the forwarding parent by solving the following optimization problem,

$$
\begin{array}{ll}
\underset{j \in \Gamma_{i}}{\operatorname{minimize}} & P_{t} Q_{j}+P_{r}\left(Q_{j}-\lambda_{j}\right) \\
\text { subject to } & R_{i, j} \cdot R(j) \geq R_{\min },
\end{array}
$$

where $P_{t}$ is the power consumption in transmission, and $P_{r}$ is the power consumption in reception, and $R_{\min }$ is the constraint on the reliability required by the application. The cost function in Eq. (V.1) is the sum of the cost for transmitting the total traffic $Q_{j}$ and cost for receiving traffic generated by children nodes $\left(Q_{j}-\lambda_{j}\right)$. The metric provides load balancing in terms of generated and forwarded traffic. As far as the implementation of this metric is concerned, node $V_{i}$ needs only local information about its own forwarded traffic $Q_{i}$, and the generated and forwarded traffic from each candidate destination, which is available through the exchange of DIO messages. We recall that load balancing is achieved typically by considering node queues, as in BCP [11]. The back-pressure algorithm in [11] uses a weighted ETX cost, 


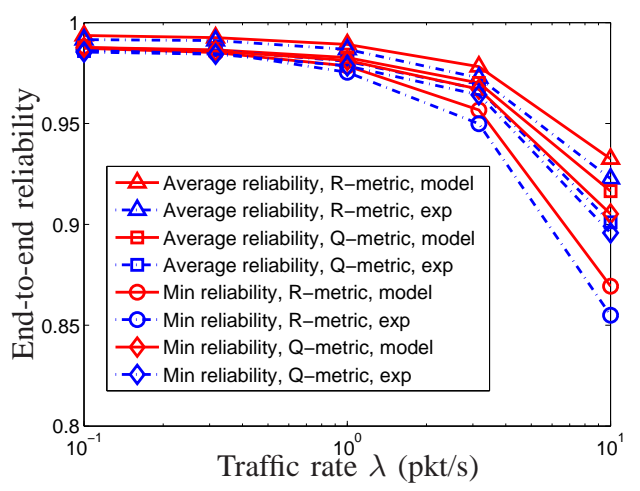

Fig. 3. End-to-end reliability for the multi-hop topology in Fig. 1. Average reliability is evaluated among nodes $V_{4}$ to $V_{7}$. Minimum reliability is the average reliability of the worst case path.

which includes the queue differential between transmitter and receiver. The protocol guarantees load balancing by avoiding at any time that node queues overloading. However, the backpressure metric is not able to capture the contention level when the traffic load is low (which is the case in most WSN applications in real life). In other words, BCP is efficient in saturated cases where the forwarded traffic is high. On the other hand, the $Q$-metric is able to directly measure the contention level without measuring the node queues and adapt the routing decisions accordingly, as we demonstrate through experiments in the next section.

\section{EXPERIMENTAL EVALUATION}

In this section, we present experimental results related to the performance of IETF RPL, the contention-based IEEE 802.15.4 MAC and the proposed enhancements. As a benchmark, we evaluate the performance of our metrics against the back-pressure algorithm proposed in [11].

We assume that nodes are deployed and connected to form a DODAG as depicted in Fig. 1. However, similar results can be derived for different topologies. The IEEE 802.15.4 protocol is implemented on a test-bed using the TelosB platform running the Contiki OS [17]. We assume that each node generates the same traffic with rate $\lambda=[0.1-10] \mathrm{pkt} / \mathrm{s}$, except $V_{2}$ that generates traffic with rate $\lambda_{2}=20 \mathrm{pkt} / \mathrm{s}$ (dominant node). We chose the unslotted MAC modality since it is one of the recommended in the IETF RPL standard. We represent then a realistic network operation in which heterogeneous traffic conditions are determined in the network both by a different traffic generation rate among nodes and distribution of the forwarded traffic among various routing paths.

In the first set of experiments, we validate our analytical model against experimental results of the two proposed metrics. Fig. 3 shows the end-to-end reliability vs the traffic rate as obtained by the mathematical model and the experiments when our proposed $R$-metric and $Q$-metric are used. We show the average reliability among nodes $V_{4}$ to $V_{7}$ and the minimum path reliability achieved in the network. We observe that the experimental results are very close to the analytical results. In Fig. 3, the minimum reliability for the $R$-metric is achieved for the path that includes $V_{4}$ and $V_{1}$. The reliability with the $Q$-metric does not vary significantly in the paths and the minimum reliability is only slightly lower than the average reliability and, furthermore, it is greater than the minimum reliability for the $R$-metric. In our experimental evaluation, the gap in the minimum reliability is around 5\% for $\lambda=10 \mathrm{pkt} / \mathrm{s}$ and it increases as the traffic rate increases. We notice that, even though the $R$-metric achieves the best average performance from a network perspective, the $Q$-metric is preferable if a guaranteed reliability is required for all paths in the network (which is desired by many control applications).

In Fig. 4, we show the end-to-end reliability of each node. We compare $R$-metric, $Q$-metric, and back-pressure. The $R$ metric guarantees high reliability for the dominant node $V_{2}$ which forwards most of the traffic in the network. However, the reliability of $V_{4}$ is compromised, since the path that includes $V_{4}$ and $V_{1}$ suffers of high level of contention from $V_{2}$. The $Q$-metric guarantees the reliability constraint in $V_{4}$ and outperforms the back-pressure metric. When using backpressure routing, we noticed frequent switches in the parent node selection. Nodes choose their parent on a packet base, by looking at the current queues. However, due to the unsaturated traffic, the value of the queues oscillates between 0 and 1 among the nodes at each transmission, with sporadic cases in which the queues are affected by the unbalanced traffic. The frequent parent switching in the back-pressure routing determines an increase of the traffic due to high number of DIO message transmissions that affects the reliability.

In Fig. 5, we show the average power consumption of each node. The power consumption is calculated by considering the sum of the contributions in transmission, reception, idlelistening, and carrier sensing for each node. By choosing the dominant node $V_{2}$ as forwarder, the $R$-metric determines an unbalanced energy consumption. Node $V_{2}$ has a power consumption up to $6 \mathrm{~mW}$, while the rest of the network operates between $0.5 \mathrm{~mW}$ and $1 \mathrm{~mW}$. With the $Q$-metric, the power consumption is more balanced among nodes and the maximum consumption, which is crucial for the network lifetime, decreases of at least a factor 2 respect to the $R$ metric. The back-pressure routing present a reduction of the maximum energy consumption with respect to the $R$-metric. However, the dominant node $V_{2}$ consumes $70 \%$ more power compared to the $Q$-metric.

We report now the results of the mathematical tool for parameter selection, by considering the multi-hop topology in Fig. 1. In the tool, we include the analytical model of the IEEE 802.15.4 MAC, derived in [15], and the analysis of the interaction with IETF RPL developed in this paper. The output of the tool is defined as the protocol and the set of MAC-routing parameters that maximize the network lifetime for certain reliability and delay constraints imposed by the application to all nodes.

We consider the unslotted IEEE 802.15.4 MAC and we let the protocol selection mechanism choose the initial backoff exponent $m_{0}=[3-8]$, the maximum backoff exponent $m_{b}=\left[m_{0}-8\right]$, and the maximum number of backoffs $m=[0-4]$. Moreover, we apply RPL and we let the mechanism choose between $R$-metric and $Q$-metric. The $Q$-metric is always preferred to the $R$-metric, whenever the solution is feasible. This result agrees with analysis and experiments presented above, since the constraints are for all nodes, and the objective is the minimization of the energy consumption 


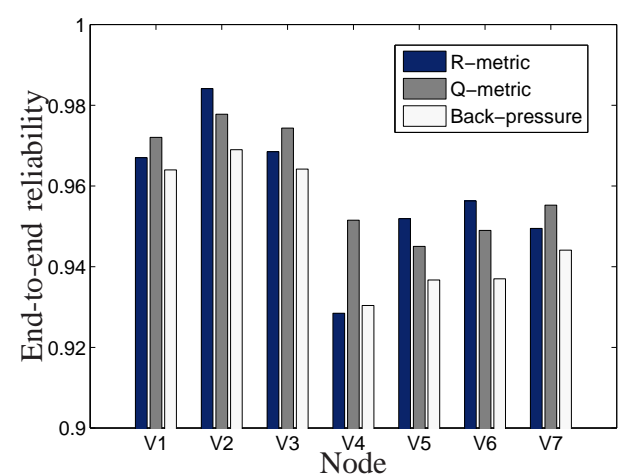

Fig. 4. End-to-end node reliability for the multi-hop topology in Fig. 1, by fixing $\lambda_{i}=5 \mathrm{pkt} / \mathrm{s}$ for $i \neq 2$ and $\lambda_{2}=20 \mathrm{pkt} / \mathrm{s}$.

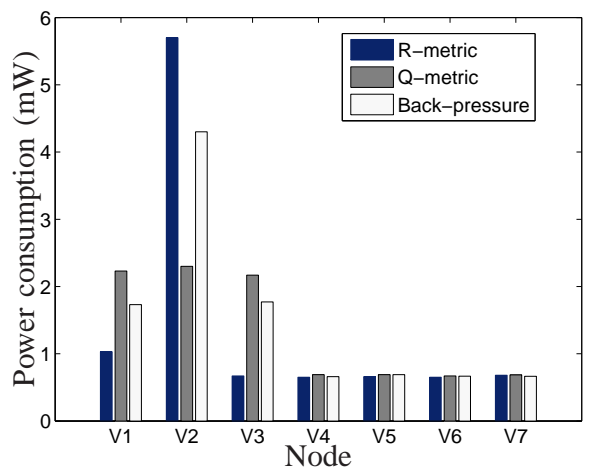

Fig. 5. Average node power consumption for the multi-hop topology in Fig. 1, by fixing $\lambda_{i}=5 \mathrm{pkt} / \mathrm{s}$ for $i \neq 2$ and $\lambda_{2}=20 \mathrm{pkt} / \mathrm{s}$.

of the dominant node. In general, an increase of the MAC parameters determines an increase in the energy consumption and in the delay. However, the reliability increases too. For a reliability constraint above $90 \%$, the optimal solution is obtained by increasing both the number of backoffs $m$ and the backoff windows $m_{0}$ and $m_{b}$. The node energy consumption associated to $m_{0}=3, m_{b}=3$, and $m=0$ is about $20 \%$ lower than the consumption with default parameters $m_{0}=3$, $m_{b}=8$, and $m=4$. In addition, as we showed in Fig. 5, the maximum energy consumption is halved as we choose the $Q$-metric over the $R$-metric. Therefore, by optimally selecting routing metric and MAC parameters according to the reliability and delay constraints, it is possible to obtain a significant impact on the performance.

\section{CONCLUSIONS}

In this paper, a mathematical description to characterize the complex inter-dependence between MAC and routing protocols in LLNs was presented. Moreover, a simple framework for joint optimization of the MAC and the routing layer parameters was proposed to enhance the existing standards. Specifically, novel metrics that take into account the dynamic behavior of the MAC and routing layers: $R$-metric and $Q$-metric were introduced. A comparison of the existing ETX metric with the reliability metric ( $R$-metric), which considers both the level of contention and the protocol parameters, was performed. Considering that a minimum reliability is required for all the nodes, or the main objective is the lifetime of the network, the $Q$-metric was proposed. Extended experiments where the

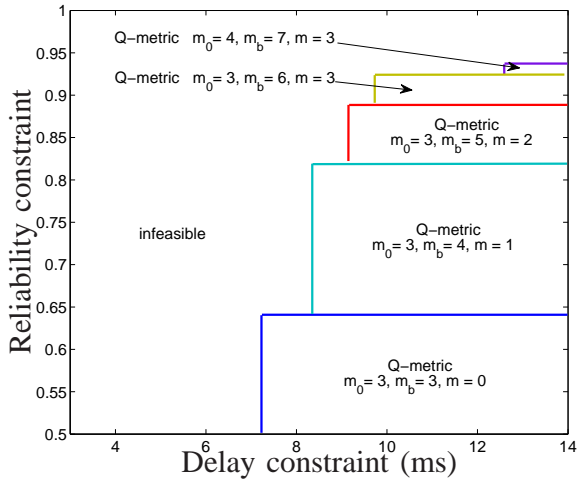

Fig. 6. IEEE 802.15.4 MAC and RPL parameters selection for the multi-hop topology in Fig. 1 by fixing $\lambda_{i}=5 \mathrm{pkt} / \mathrm{s}$ for $i \neq 2$ and $\lambda_{2}=20 \mathrm{pkt} / \mathrm{s}$.

proposed metrics were compared to the existing back-pressure routing supported the mathematical analysis.

\section{REFERENCES}

[1] J. Hui and D. Culler, "IPv6 in low-power wireless networks," Proceedings of the IEEE, vol. 98, no. 11, pp. $1865-1878,2010$.

[2] IEEE 802.15.4 standard: Wireless Medium Access Control and Physical Layer Specifications for Low-Rate Wireless Personal Area Networks, IEEE, 2006, http://www.ieee802.org/15/pub/TG4.html.

[3] ZigBee/802.15.4 Energizes the Internet of Things - A Market Dynamics report, ON World Research, 2011, http://onworld.com/zigbee.

[4] Routing Over Low power and Lossy networks, Internet Engineering Task Force (IETF), http://www.ietf.org/dyn/wg/charter/roll-charter.html.

[5] N. F. Timmons and W. G. Scanlon, "Analysis of the performance of IEEE 802.15.4 for medical sensor body area networking," in IEEE International Conference on Sensor and Ad hoc Communications and Networks, 2004.

[6] P. Park, P. Di Marco, C. Fischione, and K. H. Johansson, "Modeling and optimization of the IEEE 802.15.4 protocol for reliable and timely communications," IEEE Transactions on Parallel and Distributed Systems, vol. 24, no. 3, pp. 550-564, 2013.

[7] S. C. Ergen, P. Di Marco, and C. Fischione, "MAC protocol engine for sensor networks," in IEEE Global Communications Conference, 2009.

[8] M. Zimmerling, F. Ferrari, L. Mottola, T. Voigt, and L. Thiele, "pTunes: runtime parameter adaptation for low-power MAC protocols," in ACM/IEEE International Conference on Information Processing in Sensor Networks, 2012.

[9] D. De Couto, D. Aguayo, J. Bicket, and R. Morris, "A high-throughput path metric for multi-hop wireless routing," in ACM Conference on Mobile Computing and Networking, 2003.

[10] J. Tripathi, J. de Oliveira, and J. Vasseur, "A performance evaluation study of RPL: Routing protocol for low power and lossy networks," in IEEE Conference on Information Sciences and Systems, 2010.

[11] S. Moeller, A. Sridharan, B. Krishnamachari, and O. Gnawali, "Routing without routes: the backpressure collection protocol," in ACM/IEEE International Conference on Information Processing in Sensor Networks, 2010.

[12] E. Ghadimi, O. Landsiedel, P. Soldati, and M. Johansson, "A metric for opportunistic routing in duty cycled wireless sensor networks," in IEEE International Conference on Sensor and Ad hoc Communications and Networks, 2012.

[13] B. Pavković, F. Theoleyre, and A. Duda, "Multipath opportunistic RPL routing over IEEE 802.15.4," in ACM Conference on Modeling and Simulation of Wireless and Mobile Systems, 2011.

[14] M. Akhavan, T. Watteyne, and A. Aghvami, "Enhancing the performance of RPL using a receiver-based MAC protocol in lossy WSNs," in International Conference on Telecommunications, 2011.

[15] P. Di Marco, P. Park, C. Fischione, and K. H. Johansson, "Analytical modeling of multi-hop IEEE 802.15.4 networks," IEEE Transactions on Vehicular Technology, vol. 61, no. 7, pp. 3191-3208, 2012.

[16] C. Gomez, A. Boix, and J. Paradells, "Impact of LQI-based routing metrics on the performance of a one-to-one routing protocol for IEEE 802.15.4 multihop networks," EURASIP Journal on Wireless Communication Networks, pp. 6:1-6:20, 2010.

[17] Contiki Operating System, SICS, http://www.sics.se/contiki/aboutcontiki.html. 